\title{
A Flexible Method for Envelope Estimation in Empirical Mode Decomposition
}

\author{
Yoshikazu Washizawa ${ }^{1}$, Toshihisa Tanaka ${ }^{2,1}$, \\ Danilo P. Mandic ${ }^{3}$, and Andrzej Cichocki ${ }^{1}$ \\ ${ }^{1}$ Brain Science Institute, RIKEN, \\ 2-1, Hirosawa, Wako-shi, Saitama, 351-0198, Japan \\ \{washizawa, cia\}@brain.riken.jp \\ ${ }^{2}$ Department of Electrical and Electronic Engineering, Tokyo University of \\ Agriculture and Technology, \\ 2-24-16, Nakacho, Koganei-shi, Tokyo, 184-8588, Japan \\ tanakat@cc.tuat.ac.jp \\ ${ }^{3}$ Department of Electrical and Electronic Engineering, Imperial College London, \\ SW7 2BT, United Kingdom \\ d.mandic@imperial.ac.uk
}

\begin{abstract}
A flexible and efficient method for finding the envelope within the empirical mode decomposition (EMD) is introduced. Unlike the existing (deterministic) spline based strategy, the proposed envelope is a result of an optimisation precess and sought as a minimum of a quadratic cost function. A closed form solution of this optimisation problem is obtained and it is shown that by choosing free parameters, we can fine-tune the frequency resolution or the number of intrinsic mode functions (IMFs) as well as the shape of the envelopes. Computer simulations on both the synthetic and real-world electro-encephalogram (EEG) data support the analysis.
\end{abstract}

\section{Introduction}

The empirical mode decomposition (EMD) proposed by Huang et. al. in 1998 [1] is a technique for the analysis of non-linear and non-stationary signals in the time-frequency domain and its applications are manifold. EMD decomposes a signal $x(t)$ into its components called intrinsic mode functions (IMFs) $c_{i}(t)$, $i=1,2, \ldots n$, and the residual $r(t)$, in the following way:

$$
x(t)=\sum_{i=1}^{n} c_{i}(t)+r(t),
$$

The idea behind this approach is that every IMF has a very narrow frequency band all time, which allows us to produce a time-frequency spectrum called Hilbert-Huang $(\mathrm{HH})$ spectrum by using the Hilbert transform (for more details, see [2]). The spectrum is sharp and clear curves unlike the Wavelet or short time Fourier spectrum. As a result, to so defined spectrum exhibits well defined 
spectral components. EMD needs no a priori knowledge on the signal and as such belongs to the class of Exploratory Data Analysis techniques [3].

EMD is totally characterized by so called "upper" and "lower" envelopes of an input signal. Despite the enormous interest in this technique by the ocean modeling and each sciences research communities, and its huge potential in signal processing, little attention has been paid to and "optimal" choice of these envelopes, and instead the originally proposed spline based techniques have been employed [1. Well-known interpolations of maxima or minima such as cubic spline are often used [1. The so-generated EMD with conventional interpolations automatically determine the number of IMFs, or "the resolution of frequency." Therefore a practical problem is how to control this "frequency resolution."

To solve this problem, we propose a class of envelopes for EMD which minimizes a quadratic penalty cost function involving cost parameters, which allows us to control the bandwidth of IMFs. Since these parameters are weight coefficients in the frequency-domain. This enables a very convenient adjustment of the frequency resolution. In practical terms, if we assume wider bandwidth, fewer IMFs are needed, whereas for narrower bandwidths, we obtain more IMFs, this way can control the number of IMFs and more suitable time-frequency spectrum depending on an application.

\section{Empirical Mode Decomposition}

EMD decomposes a given signal $x$ into a number of IMFs, which have the following properties:

1. Along the signal, the number of extrema and the number of zero crossings must either be equal or differ at most by one;

2. At any point, the mean value of the envelope defined by the local maxima and the envelope defined by the local minima is zero.

Huang et. al. showed [1] that such an IMF has a very narrow frequency band. Signals such as AM, FM, AM-FM are a natural candidate for an IMF.

For given signal $x$, EMD decomposes it as in eq. (11) by the so called sifting process given by:

1. Let $\mathcal{I}$ be a index set of IMFs. Initialize $\mathcal{I}=\phi$ (empty set).

2. While $\left(x-\sum_{i \in \mathcal{I}} c_{i}\right)$ has extremum,

(a) Set $h=x-\sum_{i \in \mathcal{I}} c_{i}$

(b) While $h$ is not IMF.

- Detect local maxima and minima. Interpolate them by cubic splines. Let $u$ and $l$ be respectively the upper and the lower envelope.

- Set $h \leftarrow h-\frac{1}{2}(u+l)$

(c) Add $h$ to the set of IMF. 
The stopping criterion of IMF in 2.(b) is a crucial problem which in [1] was solved by using the standard deviation (SD):

$$
S D=\sum_{t} \frac{\left|h_{\text {old }}(t)-h_{\text {new }}(t)\right|^{2}}{h_{\text {old }}^{2}(t)} .
$$

In [1] a typical heuristic value for this stopping criterion was set to a value between 0.2 and 0.3 .

\section{Definition of Envelopes by Quadratic Penalty Function}

Let $f$ be a signal to be decomposed, $\left\{t_{i}^{u}\right\}$ and $\left\{t_{j}^{l}\right\}\left(i=1, \ldots, N_{u}, j=1, \ldots, N_{l}\right)$ be sets of time instances of maxima and minima, respectively.

Without loss in generality, we shall consider the upper envelope (the lower envelope can be analyzed the same way). The problem to find $u$ can be formulated as a classical linear estimation problem from a set of samples $\left\{f\left(t_{i}^{u}\right)\right\}$. Specifically, we assume that the sampling of $u$ was performed as,

$$
\sum_{i=1}^{N_{u}}\left(e_{i} \otimes \overline{k\left(\cdot, t_{i}^{u}\right)}\right) u=\left[\begin{array}{c}
f\left(t_{1}^{u}\right) \\
\vdots \\
f\left(t_{N_{u}}^{u}\right)
\end{array}\right]
$$

where $k(\cdot, \cdot)$ is the reproducing kernel of the signal space, $\left(\cdot \otimes^{\bar{*}}\right)$ is the NeumannShatten product defined by $(a \otimes \bar{b}) c=\langle c, b\rangle a$, and $e_{i}$ is the $i$-th natural basis in $\mathbb{R}^{N_{u}}$. Let $A=\sum_{i=1}^{N_{u}}\left(e_{i} \otimes \overline{k\left(\cdot, t_{i}^{u}\right)}\right)=A$ and $f_{v}=\left[\begin{array}{c}f\left(t_{1}^{u}\right) \\ \vdots \\ f\left(t_{N_{u}}^{u}\right)\end{array}\right]$. Then (3) is reduced to $A u=f_{v}$. If $u$ is a discrete consisting of $N$ sample, then $A \in \mathbb{R}^{N_{u} \times N}$ is explicitly expressed as

$$
A=\sum_{i=1}^{N_{u}}\left(e_{i} \hat{e}_{t_{i}^{u}}^{\top}\right)
$$

where $\hat{e}_{i}$ is an $i$-th natural basis in $\mathbb{R}^{N}$.

The upper envelope is given by a solution of the above linear formula to yield

$$
u=A^{\dagger} f_{v}+w, \quad w \in \mathcal{N}(A),
$$

where $A^{\dagger}$ is the Moore-Penrose generalized inverse of $A$ and $\mathcal{N}(A)$ is the null space of $A$. If $A A^{*}=I, A$ is a partial isometry matrix i.e., $A^{\dagger}=A^{\top}$. We still have an ambiguity on $u$, that is $w$ an arbitrary vector. The following discussion is devoted to determine the unique $w$.

Let $\mathcal{F}$ be a Fourier transform operator and $U$ be a Fourier transform of $u$, that is

$$
U=\mathcal{F} u=\sum_{t=0}^{N_{u}-1} u(t) \overline{\exp (i \xi t)}
$$


We can now introduce the following cost function,

$$
J=\sum_{i=1}^{N} U\left(\xi_{i}\right) L \overline{U\left(\xi_{i}\right)}=\langle U, L U\rangle,
$$

where $L$ is a self adjoint cost operator defined as,

$$
L=\operatorname{diag}(\boldsymbol{\rho})=\left[\begin{array}{lll}
\rho_{1} & & 0 \\
& \ddots & \\
0 & & \rho_{N}
\end{array}\right] .
$$

and $\left\{\xi_{i}\right\}_{i=1}^{N}$ is a set of discretized frequencies such that $\xi_{i}<\xi_{j}$ if $i<j$.

The underlying idea behind this cost function is that an envelope should be smooth, i.e., its frequency spectrum should be biased to its lower part. Different definitions of $\rho_{i}$ result in different envelopes. For example, $\boldsymbol{\rho}$ can be given as follows:

1. $\boldsymbol{\rho}=(\underbrace{0 \ldots 0}_{n} \underbrace{1 \ldots 1}_{N-2 n} \underbrace{0 \ldots 0}_{n})$

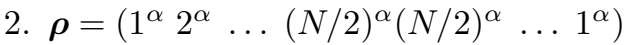

3. $\boldsymbol{\rho}=\left(e^{\alpha} e^{2 \alpha} \ldots e^{N \alpha / 2} e^{N \alpha / 2} \ldots e^{\alpha}\right)$

Intuitively, the case 1 suggests that high frequency components of an envelope are mostly suppressed. The second and third cases imply that a higher components of an envelope cost the higher because the shape of frequency spectrum tends to decays polynomially or exponentially. Various $\boldsymbol{\rho}$ can be alternated during a sifting process.

In summery, the optimization problem to be solved here is:

$$
\begin{aligned}
\min _{u} J & =\langle\mathcal{F} u, L \mathcal{F} u\rangle, \\
\text { subject to } u & =A^{\dagger} f_{v}+w, w \in \mathcal{N}(A) .
\end{aligned}
$$

Theorem 1. Optimization problem (9) is minimized when

$$
\begin{aligned}
u= & A^{\dagger} f_{v} \\
& -\left(I-A^{\dagger} A\right)\left(\left(I-A^{\dagger} A\right)^{*} \mathcal{F}^{*} L \mathcal{F}\left(I-A^{\dagger} A\right)\right)^{\dagger}\left(I-A^{\dagger} A\right)^{*} \mathcal{F}^{*} L \mathcal{F} A^{\dagger} f_{v} .
\end{aligned}
$$

(Proof). We can change the constraint to

$$
u=A^{\dagger} f_{v}+\left(I-A^{\dagger} A\right) w,
$$

without loss of generality since $\left(I-A^{\dagger} A\right)$ is the projection operator onto $\mathcal{N}(A)$ i.e, $\mathcal{N}(A)=\mathcal{R}\left(I-A^{\dagger} A\right)$.

By subsisting $u$ as in (11) to (9), we have

$$
\begin{aligned}
J= & \left\langle\mathcal{F}\left(A^{\dagger} f_{v}+\left(I-A^{\dagger} A\right) w\right), L \mathcal{F}\left(A^{\dagger} f_{v}+\left(I-A^{\dagger} A\right) w\right)\right\rangle \\
= & \left\langle\mathcal{F} A^{\dagger} f_{v}, L \mathcal{F} A^{\dagger} f_{v}\right\rangle+\left\langle w,\left(I-A^{\dagger} A\right)^{*} \mathcal{F}^{*} L \mathcal{F} A^{\dagger} f_{v}\right\rangle \\
& +\left\langle\left(I-A^{\dagger} A\right)^{*} \mathcal{F}^{*} L \mathcal{F} A^{\dagger} f_{v}, w\right\rangle+\left\langle w,\left(I-A^{\dagger} A\right)^{*} \mathcal{F}^{*} L \mathcal{F}\left(I-A^{\dagger} A\right) w\right\rangle .
\end{aligned}
$$


Then the vector which has to be optimized is changed to $w$.

Since $\left(I-A^{\dagger} A\right)^{*} \mathcal{F}^{*} L \mathcal{F}\left(I-A^{\dagger} A\right)$ is non-negative definite, $w$ is given as

$$
w=-\left(I-A^{\dagger} A\right)\left(\left(I-A^{\dagger} A\right)^{*} \mathcal{F}^{*} L \mathcal{F}\left(I-A^{\dagger} A\right)\right)^{\dagger}\left(I-A^{\dagger} A\right)^{*} \mathcal{F}^{*} L \mathcal{F} A^{\dagger} f_{v} .
$$

\section{Experiments}

Two experimental results illustrate the effectiveness and flexibility of the proposed method. We used the publicly available MATLAB codes [4]-6] to compare our proposed EMD with the conventional one.

First, to illustrate the behavior of the envelopes given in (10), we generated a synthetic signal,

$$
x(t)=\sin \left(\frac{2}{40} \pi t\right) \sin \left(\frac{2}{400} \pi t\right),
$$

where $x(t)$ is discrete signal with $t=1, \ldots, 800$. We set $\boldsymbol{\rho}=\left(\begin{array}{llllll}1^{\alpha} & 2^{\alpha} \ldots 400^{\alpha} 400^{\alpha} \ldots 1^{\alpha}\end{array}\right)$. The envelops obtained from the proposed method with variable $\alpha$ are shown in Figure 1 It can be observed that a larger $\alpha$ yields a smoother envelope. We can also observe from Figure 1 the various shapes of the mean of upper and lower envelopes. When $\alpha=1$, variation in the mean signal is considerable, which implies that the sifting process removes high frequency components. In that case, the bandwidth of IMF is narrow, and the number of IMFs is large.

Next, we applied the proposed method to a real biomedical signal. We used a single channel of electroencephalogram (EEG). The experiments was conducted by Rutkowski et. al. within the so-called Steady State Visual Evoked Potential (SSVEP) mode [7. Within this framework, the subjects are asked to focus their

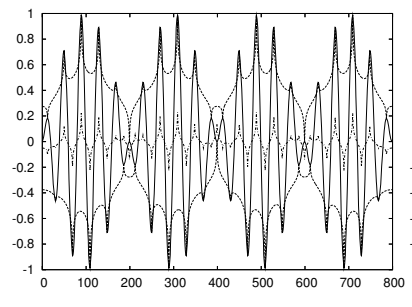

$\alpha=1$

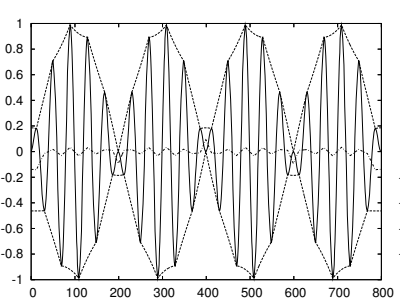

$\alpha=2$

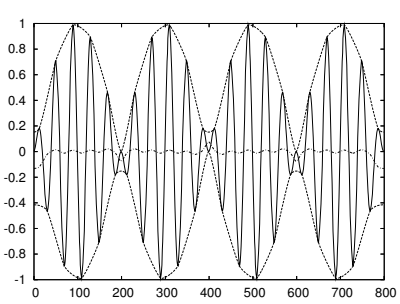

$\alpha=2.5$

Fig. 1. Original signals, envelopes and means of envelopes

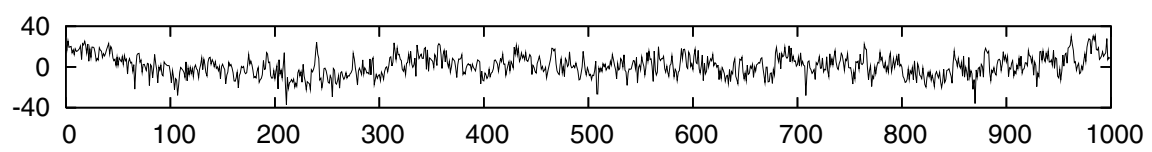

Fig. 2. A single channel of EEG signal 


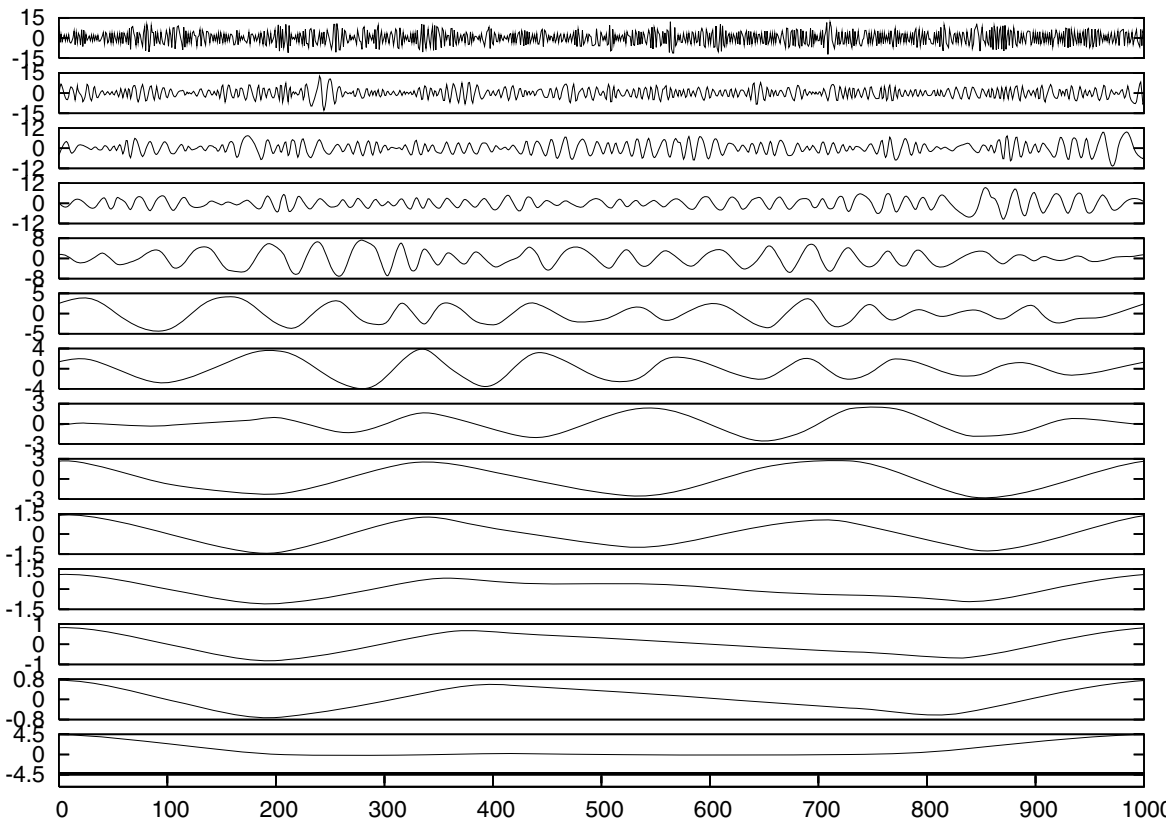

Fig. 3. IMFs of the EEG signal (Proposed method $\alpha=3$ )

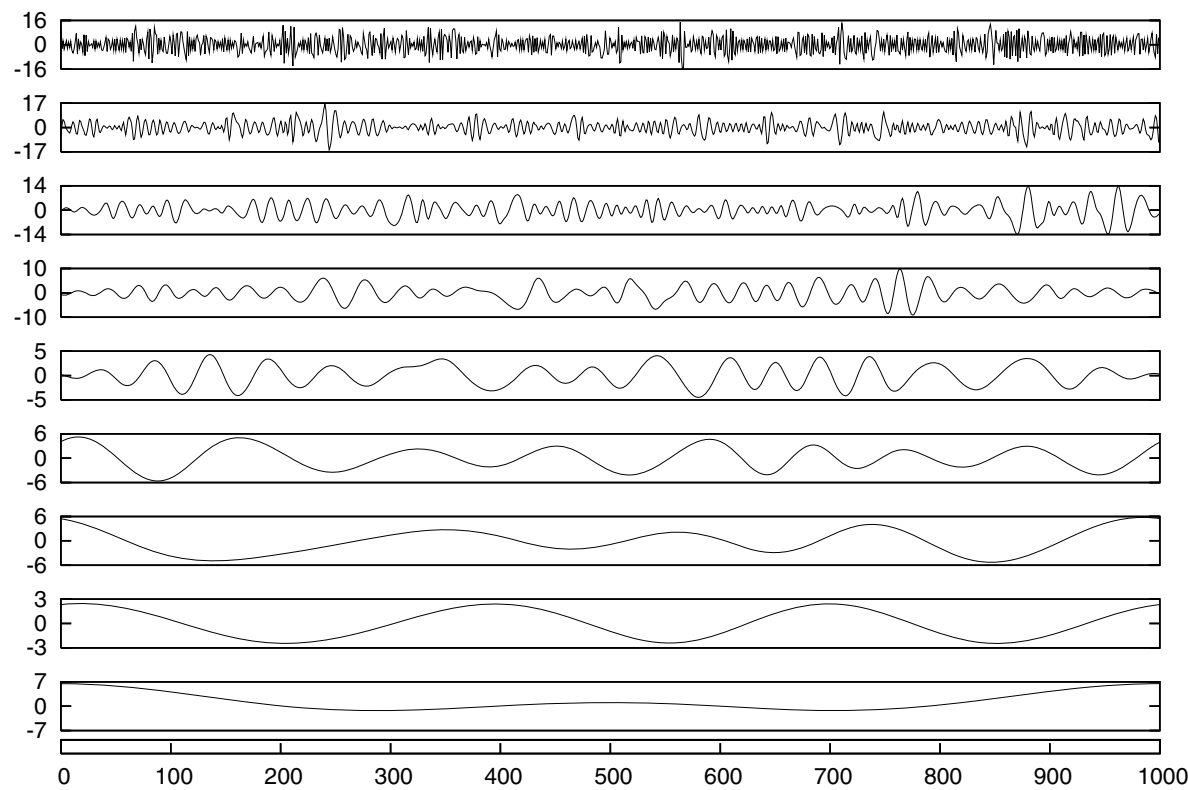

Fig. 4. IMFs of the EEG signal (Proposed method $\alpha=5$ ) 


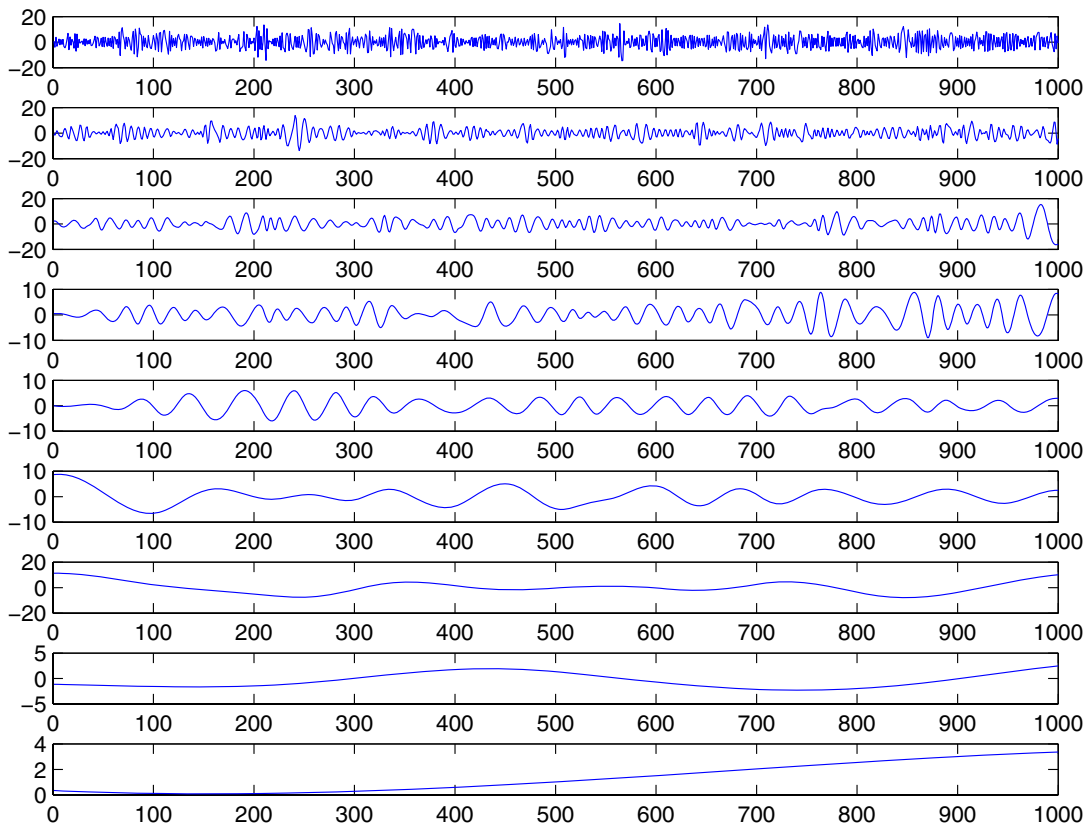

Fig. 5. IMFs of the EEG signal (Conventional method)

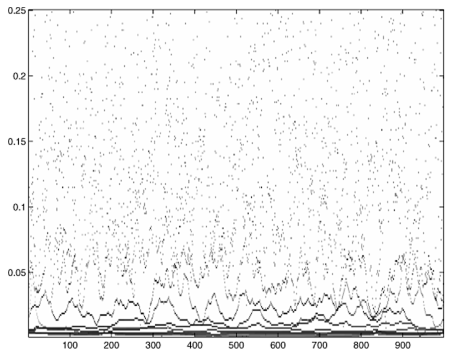

$\alpha=3$

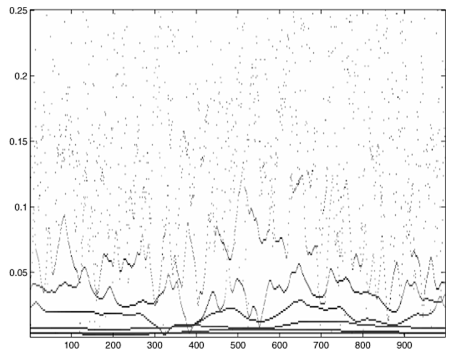

$\alpha=5$

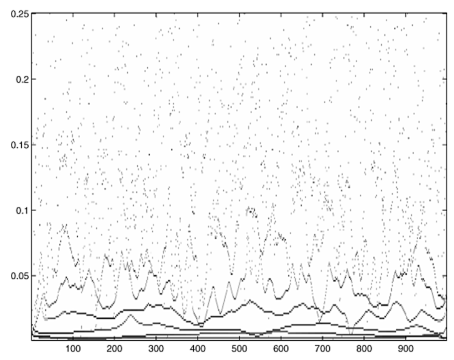

Conventional EMD

Fig. 6. Comparison of Hilbert-Huang spectra (Vertical axis: normalized frequency, horizontal axis: sample) 
attention on simple flashing stimuli, whose frequency is $1 / 26 \mathrm{~Hz}$. The stimuli is given from one second after (205 sample). The EEG signal under this subject was recorded by an A/D converter with a sampling rate of $2048 \mathrm{~Hz}$ and a bit depth of $24 \mathrm{bits}$, followed by a down-sampler to $204.8 \mathrm{~Hz}$. Figure 2 shows this signal.

We tested three values of $\alpha, \alpha=3,5,7$, with $\boldsymbol{\rho}=\left(1^{\alpha} 2^{\alpha} \ldots 500^{\alpha} 500^{\alpha} \ldots 1^{\alpha}\right)$. Figures 3, 4 and 5 compare the IMFs obtained by the proposed method with those from the conventional EMD with cubic spline. When $\alpha=3$, the number of IMFs was 14 , while when $\alpha=5$, the number of IMFs was 9 . The number of IMFs of conventional EMD was 8. These results imply that to tune parameter $\boldsymbol{\rho}$, we can control the number of IMFs and the bandwidth of IMF.

Figure 6 shows the Hilbert-Huang spectrum of the EEG signal. For $\alpha=3$ observe a dense and higher resolution spectrum, while for $\alpha=5$, the spectrum is rough and with lower resolution.

\section{Discussion and Conclusions}

A new approach for the derivation of the envelopes within the empirical mode decomposition (EMD) method has been proposed. It allows us to control the frequency bandwidth of IMFs, their number, and the resolution of the HilbertHuang spectrum.

In the proposed method, to obtain the solution, we have to compute a generalized inverse to $\mathbb{R}^{N}$ of which dimension equals to the number of samples. It requires heavy computation for the inverse, which is a subject of our follow-up work. In our experiments, we used fixed cost parameter $\boldsymbol{\rho}$. Indeed, it can be changed during sifting process. Various $\boldsymbol{\rho}$ will also give different and interesting results. For example, by changing $\boldsymbol{\rho}$, higher resolution in low frequency parts in Hilbert-Huang spectrum could be obtained. Efficient and useful methods to alter $\boldsymbol{\rho}$ would be addressed in the near future.

\section{References}

1. Huang, N. E., Shen, Z., Long, S. R., Wu, M. C., Shih, H. H., Zheng, Q., Yen, N-C., Tung, C. C., Liu, H. H.: Empirical Mode Decomposition Method and the Hilbert Spectrum for Non-stationary Time Series Analysis. Proc. Roy. Soc. London, A454, (1998), 903-995.

2. Hahn, S. L.,: Hilbert Transforms in Signal Processing. Artech House Publishers, 1996.

3. Tukey, J. W.,: Exploratory data analysis. Addison-Wesley, 1977.

4. The time-frequency toolbox. http://tftb.nongnu.org/

5. Rilling, G., Flandrin, P., Gonçalvés, P.: MATLAB codes for EMD. http:// perso.ens-lyon.fr/patrick.flandrin/emd.html

6. Lambert, M., Engroff, A., Dyer, M., Byer, B.: MATLAB codes for EMD. http://www.owlnet.rice.edu/ elec301/Projects02/empiricalMode/code.html

7. Rutkowski, T. M., Vialatte, F., Cichocki, A., Mandic, D. P., Barros, A. K.: Auditory Feedback for Brain Computer Interface Management An EEG Data Sonification Approach. Proc. of 10th International Conference on Knowledge-Based \& Intelligent Information \& Engineering Systems (KES2006) in this volume. 